\title{
Improved Discrete Techniques of Time-Delay and Order Estimation for Large-Scale Interconnected Nonlinear Systems
}

\author{
Mourad Elloumi and Samira Kamoun \\ National School of Engineers of Sfax (ENIS), Laboratory of Sciences and Techniques of \\ Automatic Control \& Computer Engineering (Lab-STA), University of Sfax, BP 1173, 3038 Sfax, Tunisia
}

Correspondence should be addressed to Mourad Elloumi; mourad.elloumi@yahoo.fr

Received 6 April 2017; Revised 13 July 2017; Accepted 19 July 2017; Published 12 September 2017

Academic Editor: Huanqing Wang

Copyright (C) 2017 Mourad Elloumi and Samira Kamoun. This is an open access article distributed under the Creative Commons Attribution License, which permits unrestricted use, distribution, and reproduction in any medium, provided the original work is properly cited.

\begin{abstract}
The selection of a suitable model structure is an essential step in system modeling. Model structure is defined by determining the class, the time delay, and the model order. This paper proposes improved structural estimation procedures for large-scale interconnected nonlinear systems which are composed of a set of interconnected Single-Input Single-Output (SISO) Hammerstein structures and described by discrete-time stochastic models with unknown time-varying parameters. An extensive Determinant Report (DR) algorithm is developed to determine the order of the process. An improved discrete-time technique based on Recursive Extended Least Squares with Varying Time (RELSVT) delay method is proposed to estimate the time delays of the considered system. The developed theoretical analysis and simulation results prove the validity and performance of the proposed algorithms.
\end{abstract}

\section{Introduction}

In recent years, several theoretical and practical researches dealing with different themes, like the modeling, the identification, and the control for complex systems, have been developed in the literature [1-22]. These works preoccupied more particularly the large-scale systems [1-14], the nonlinear systems [1-22], and the nonstationary systems [12-14]. The class of large-scale systems can be found in different fields as transportation processes, power processes, communication networks, space structures, and others.

Note that the study of the problems related to the description of a dynamic system by a mathematical model has been always a main objective to be met in different fields of science and automatic engineering, more particularly in the identification process. In this case, several automation engineers are interested in the study of this problem and various works dealing with this topic are developed and published in the literature. It is important to note that, before applying the identification methods, it is necessary to have a priori information about the structure of the process to be estimated. However, the order and the time delay of the process may be unknown in a practical estimation problem. Therefore, the estimation of these structural parameters is a fundamental problem. Add to that, in automatic control and signal processing applications, the time-delay and the order estimation of a certain process can be a means to achieve a good model for the control design. In this instance, various structural estimation methods have been proposed in the literature [15-28].

The purpose of this framework is to develop two procedures which can be applied to the class of large-scale interconnected systems with unknown time-varying parameters, in order to formulate the structure estimation problem. We focused on the dynamic large-scale stochastic systems comprised of several SISO interconnected nonlinear subsystems and represented by an interconnection of $N$ Hammerstein structures with unknown parameters.

The rest of this article was organized as follows. The second section describes the dynamics of large-scale interconnected systems by stochastic Hammerstein mathematical models with unknown time-varying parameters. Section 3 
details the formulation of the structural identification problem of interconnected nonlinear systems where two iterative procedures are proposed. The main purpose of these developed algorithms is to estimate the order model and the time delay for this class of dynamical systems. Numerical simulation examples are treated to validate our developed analytical results. Conclusions are delineated in Section 4.

\section{Preliminary Study}

A dynamic large-scale interconnected system is characterized not only by a large number of dynamics, but also by input and output signals. It can be presented by a mathematical model composed of a large number of equations, which are connected between themselves and contain several signals and parameters. In the literature, several presentations are used to describe the dynamics of this class of systems. We can cite input-output discrete models [11], continuous statespace models $[1,2]$, and block-oriented mathematical models $[10,12-14]$. In this framework, we consider the class of largescale systems which consist of several interconnected Hammerstein subsystems operating in a stochastic environment with unknown time-varying parameters and described by the following dynamic equation:

$$
\begin{aligned}
A_{i}\left(q^{-1}, k\right) y_{i}(k)= & q^{-d_{i}} B_{i}\left(q^{-1}, k\right) h_{i}^{u_{i}}(k) \\
& +\sum_{j=1, j \neq i}^{N} q^{-d_{i j}} B_{i j}\left(q^{-1}, k\right) h_{j}^{u_{j}}(k) \\
& +\sum_{j=1, j \neq i}^{N} q^{-t_{i j}} A_{i j}\left(q^{-1}, k\right) h_{j}^{y_{j}}(k) \\
& +C_{i}\left(q^{-1}\right) e_{i}(k),
\end{aligned}
$$

in which the nonlinear functions $h_{i}^{u_{i}}(k), h_{j}^{u_{j}}(k)$, and $h_{j}^{y_{j}}(k)$ are approximated by the following polynomials:

$$
\begin{aligned}
& h_{j}^{u_{i}}(k)=\alpha_{i, 1} u_{i}(k)+\alpha_{i, 2} u_{i}^{2}(k)+\cdots+\alpha_{i, p_{1}} u_{i}^{p_{1}}(k), \\
& h_{j}^{u_{j}}(k)=\beta_{j, 1} u_{j}(k)+\beta_{j, 2} u_{j}^{2}(k)+\cdots+\beta_{j, p_{2}} u_{j}^{p_{2}}(k), \\
& h_{j}^{y_{j}}(k)=\gamma_{j, 1} y_{j}(k)+\gamma_{j, 2} y_{j}^{2}(k)+\cdots+\gamma_{j, p_{3}} y_{j}^{p_{3}}(k),
\end{aligned}
$$

where $q^{-1}$ is the backward shift operator; $y_{i}(k)$ and $u_{i}(k)$ represent, respectively, the output and the input of each interconnected nonlinear system $S_{i} ; d_{i}, d_{i j}$, and $t_{i j}$ correspond to the time delays of the system $S_{i}$ and the other interaction systems $S_{j}, i, j=1, \ldots, N, j \neq i ; \alpha_{i, r_{1}}, \beta_{j, r_{2}}$, and $\gamma_{j, r_{3}}$ are unknown constant parameters of the nonlinear functions; and $A_{i}\left(q^{-1}, k\right), B_{i}\left(q^{-1}, k\right), B_{i j}\left(q^{-1}, k\right), A_{i j}\left(q^{-1}, k\right)$, and $C_{i}\left(q^{-1}\right)$ are described by

$$
\begin{aligned}
& A_{i}\left(q^{-1}, k\right)=1+a_{i, 1}(k) q^{-1}+\cdots+a_{i, n_{A_{i}}}(k) q^{-n_{A_{i}}}, \\
& B_{i}\left(q^{-1}, k\right)=b_{i, 1}(k) q^{-1}+\cdots+b_{i, n_{B_{i}}}(k) q^{-n_{B_{i}}}, \\
& B_{i j}\left(q^{-1}, k\right)=b_{i j, 1}(k) q^{-1}+\cdots+b_{i j, n_{i j}}(k) q^{-n_{B_{i j}}}
\end{aligned}
$$

$$
\begin{gathered}
A_{i j}\left(q^{-1}, k\right)=1+a_{i j, 1}(k) q^{-1}+\cdots+a_{i j, n_{A_{i j}}}(k) q^{-n_{A_{i j}}}, \\
C_{i}\left(q^{-1}\right)=1+c_{i, 1} q^{-1}+\cdots+c_{i, n_{C_{i}}} q^{-n_{C_{i}}}
\end{gathered}
$$

with $i, j=1, \ldots, N, j \neq i$, and $n_{A_{i}}, n_{B_{i}}, n_{A_{i j}}, n_{B_{i j}}$, and $n_{C_{i}}$ are the orders of the polynomials $A_{i}\left(q^{-1}, k\right), B_{i}\left(q^{-1}, k\right)$, $A_{i j}\left(q^{-1}, k\right), B_{i j}\left(q^{-1}, k\right)$, and $C_{i}\left(q^{-1}\right)$, respectively.

For the sake of simplicity, in what follows, we retain that $A_{i}\left(q^{-1}, k\right), B_{i}\left(q^{-1}, k\right), A_{i j}\left(q^{-1}, k\right), B_{i j}\left(q^{-1}, k\right)$, and $C_{i}\left(q^{-1}\right)$ have an even order $n_{i}$. We assume also that the noise $\left\{e_{i}(k)\right\}$ is a sequence of independent random variables with zero mean and constant variance $\sigma_{i}^{2}$. In addition, this sequence is uncorrelated with all input and output signals. Furthermore, we can rewrite the system output in the following developed form:

$$
\begin{aligned}
y_{i}(k)= & -a_{i, 1}(k) y_{i}(k-1)-\cdots-a_{i, n_{i}}(k) y_{i}\left(k-n_{i}\right) \\
& +b_{i, 1}(k) \alpha_{i, 1} u_{i}(k-1)+b_{i, 2}(k) \alpha_{i, 1} u_{i}(k-2) \\
& +\cdots+b_{i, n_{i}}(k) \alpha_{i, 1} u_{i}\left(k-n_{i}\right)+\cdots \\
& +b_{i, 1}(k) \alpha_{i, p_{1}} u_{i}^{p_{1}}(k-1) \\
& +b_{i, 2}(k) \alpha_{i, p_{1}} u_{i}^{p_{1}}(k-2)+\cdots \\
& +b_{i, n_{i}}(k) \alpha_{i, p_{1}} u_{i}^{p_{1}}\left(k-n_{i}\right) \\
& +b_{i j, 1}(k) \beta_{j, 1} u_{j}(k-1)+\cdots \\
& +b_{i j, n_{i}}(k) \beta_{j, 1} u_{j}\left(k-n_{i}\right)+\cdots \\
& +b_{i j, 1}(k) \beta_{j, p_{2}} u_{j}^{p_{2}}(k-1)+\cdots \\
& +b_{i j, n_{i}}(k) \beta_{j, p_{2}} u_{j}^{p_{2}}\left(k-n_{i}\right) \\
& +a_{i j, 1}(k) \gamma_{j, 1} y_{j}(k-1)+\cdots \\
& +a_{i j, n_{i}}(k) \gamma_{j, 1} y_{j}\left(k-n_{i}\right)+\cdots \\
& +a_{i j, 1}(k) \gamma_{j, p_{3}} y_{j}^{p_{3}}(k-1)+\cdots \\
& +a_{i j, n_{i}}(k) \gamma_{j, p_{3}} y_{j}^{p_{3}}\left(k-n_{i}\right)+e_{i}(k) \\
& +c_{i, 1} e_{i}(k-1)+\cdots+c_{i, n_{i}} e_{i}\left(k-n_{i}\right) . \\
& +\cdots
\end{aligned}
$$

We note that some implementation difficulties are presented in the formulation of the parametric estimation problem in spite of the presence of some redundant parameters in model (4). From [12], we propose that $\gamma_{j, 1}, b_{i, 1}(k)$, and $b_{i j, 1}(k)$ are constant and known, in such a way that $\gamma_{j, 1}=b_{i j, 1}(k)=$ $b_{i, 1}(k)=1, \forall k$, in order to avoid the posed problem. In this case, each output $y_{i}(k)$ of the considered interconnected system becomes

$$
\begin{aligned}
y_{i}(k)= & -a_{i, 1}(k) y_{i}(k-1)-\cdots-a_{i, n_{i}}(k) y_{i}\left(k-n_{i}\right) \\
& +\alpha_{i, 1} u_{i}(k-1)+\alpha_{i, 1} b_{i, 2}(k) u_{i}(k-2)+\cdots \\
& +\alpha_{i, 1} b_{i, n_{i}}(k) u_{i}\left(k-n_{i}\right)+\cdots+\alpha_{i, p_{1}} u_{i}^{p_{1}}(k-1)
\end{aligned}
$$




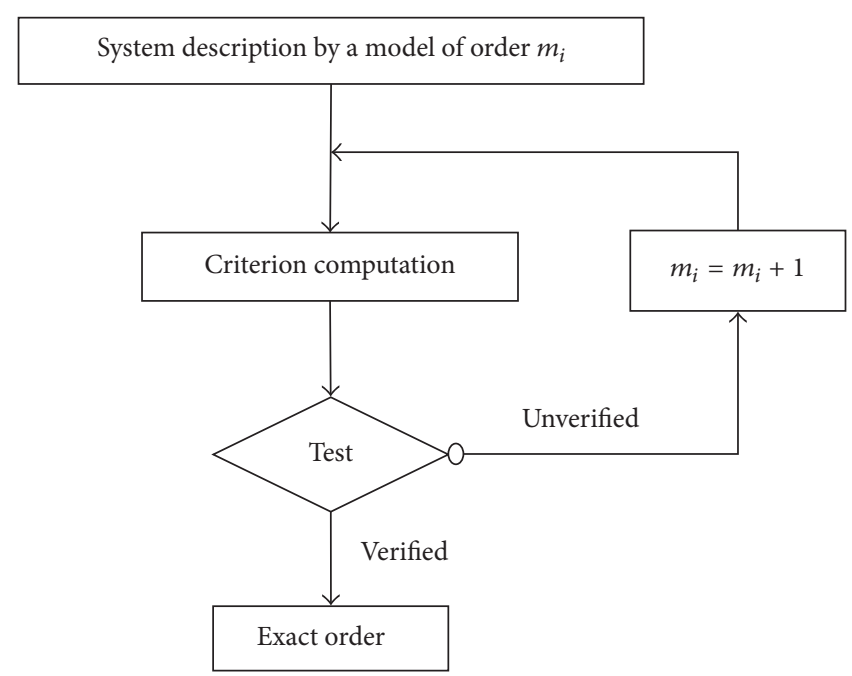

FIGURE 1: Order estimation procedure.

$$
\begin{aligned}
& +\alpha_{i, p_{1}} b_{i, 2}(k) u_{i}^{p_{1}}(k-2)+\cdots \\
& +\alpha_{i, p_{1}} b_{i, n_{i}}(k) u_{i}^{p_{1}}\left(k-n_{i}\right)+\beta_{j, 1} u_{j}(k-1) \\
& +\cdots+\beta_{j, 1} b_{i j, n_{i}}(k) u_{j}\left(k-n_{i}\right)+\cdots \\
& +\beta_{j, p_{2}} u_{j}^{p_{2}}(k-1)+\cdots \\
& +\beta_{j, p_{2}} b_{i j, n_{i}}(k) u_{j}^{p_{2}}\left(k-n_{i}\right) \\
& +a_{i j, 1}(k) y_{j}(k-1)+\cdots+a_{i j, n_{i}}(k) y_{j}\left(k-n_{i}\right) \\
& +\cdots+\gamma_{j, p_{3}} a_{i j, 1}(k) y_{j}^{p_{3}}(k-1)+\cdots \\
& +\gamma_{j, p_{3}} a_{i j, n_{i}}(k) y_{j}^{p_{3}}\left(k-n_{i}\right)+e_{i}(k) \\
& +c_{i, 1} e_{i}(k-1)+\cdots+c_{i, n_{i}} e_{i}\left(k-n_{i}\right) .
\end{aligned}
$$

The formulation of the parametric estimation problem for this class of large-scale systems, which is described by the discrete model (5), was proved by Elloumi and Kamoun [12]. In this instance, the developed parameter estimation procedure is based on the adjustable model and the prediction error method, starting from the knowledge of several measured input-output signals resulting from the considered process. In this case, we supposed that the time delays and the order model of the considered system are known. For this reason, serious errors can be obtained in the synthesis of a control design if the used order model and/or time-delay parameters are wrong.

Note that the dynamic process models are always desirable in order to provide the required design of regulators. In addition, the choice of the model structure (order and time delay) and the parameter estimation are two basic elements in the identification problem. Therefore, lots of works that used various methods to estimate the time delay and the order model have been published in the literature [15-28].

\section{Structural Estimation}

In this section, we are proposed to formulate the structural estimation problem for the class of large-scale interconnected nonlinear stochastic systems described by the mathematical model (1). The formulation of this problem can be classified into two subsections.

The first subsection seeks to determine the order of the considered process, where its dynamics are modeled by INARMAX mathematical model (5). The order estimate will be performed on the basis of an extension of DR algorithm that can be applied to this class of complex systems, whereas the formulation of the time-delay estimation problem is considered in the second subsection. In this context, an improved procedure based on RELSVT algorithm is proposed.

3.1. Order Estimation. The adequate value of the order process is one of the major preoccupations in the identification problem. Lots of testing methods have been proposed for determining the orders of discrete dynamic processes, like the Determinant Report (DR) method, the behavior of error function, polynomial approach, test of normality, and so forth [15-17]. In [15], the developed DR technique is based on the product-moment matrix containing several input-output signals measurements from the system.

In this part, the purpose is to extend this approach in order to estimate the order of large-scale interconnected nonlinear systems, which is described by the discrete-time mathematical model (1).

The organizational chart of the order estimate, represented in Figure 1, seeks to verify certain criteria after describing the system by a mathematical model with an order selected a priori $m_{i}\left(m_{i}=1,2, \ldots, m_{i \max }\right.$, where $m_{i \max }$ is the maximum order). The exact model order of the system corresponds to the value $m_{i}$ for which the calculated criterion presents a particular deviation. 
This procedure seeks to limit the number of possible orders and determine the accurate one, and this is based on the computation of the product-moment matrix with dimension $\left(\left(p_{1}+p_{2}+p_{3}+1\right) m_{i},\left(p_{1}+p_{2}+p_{3}+1\right) m_{i}\right)$, which is defined by

$$
Q_{i}\left(m_{i}\right)=\frac{1}{M_{k}} \sum_{m_{i}}^{M_{k}+m_{i}} q_{i}\left(m_{i}\right) q_{i}^{T}\left(m_{i}\right)
$$

with

$$
\begin{aligned}
q_{i}^{T} & \left(m_{i}\right)=\left[y_{i}(k-1) u_{i}(k-1) \cdots u_{i}^{p_{1}}(k-1)\right. \\
& \cdot u_{j}(k-1) \cdots u_{j}^{p_{2}}(k-1) \\
& \cdot y_{j}(k-1) \cdots y_{j}^{p_{3}}(k-1) \cdots y_{i}\left(k-m_{i}\right) \\
\cdot & u_{i}\left(k-m_{i}\right) \cdots u_{i}^{p_{1}}\left(k-m_{i}\right) \\
\cdot & u_{j}\left(k-m_{i}\right) \cdots u_{j}^{p_{2}}\left(k-m_{i}\right) \\
\cdot & \left.y_{j}\left(k-m_{i}\right) \cdots y_{j}^{p_{3}}\left(k-m_{i}\right)\right],
\end{aligned}
$$

where $M_{k}$ represents the measurements number and $m_{i}$ depicts the model order of the process, $i, j=1, \ldots, N, j \neq i$.

The matrix $Q_{i}\left(m_{i}\right)$ possesses the following properties:

$$
\operatorname{det}\left[Q_{i}\left(m_{i}\right)\right]:\left\{\begin{array}{ll}
>0 & \text { if } m_{i}<n_{i} \\
=0 & \text { if } m_{i}>n_{i} ;
\end{array} \quad i=1, \ldots, N\right.
$$

This matrix is positive definite and it is singular, if $m_{i}>n_{i}$.

For $m_{i}<n_{i}, \mathrm{DR}_{i}$ is expressed by

$$
\operatorname{DR}_{i}\left(m_{i}\right)=\left|\frac{\operatorname{det}\left[Q_{i}\left(m_{i}\right)\right]}{\operatorname{det}\left[Q_{i}\left(m_{i}+1\right)\right]}\right|
$$

with

$$
\begin{aligned}
& \operatorname{DR}_{i}\left(m_{i}\right)>0 \quad \text { if } m_{i}<n_{i}, \\
& \operatorname{DR}_{i}\left(m_{i}\right) \gg \operatorname{DR}_{i}\left(m_{i}-1\right) \quad \text { if } m_{i}=n_{i} .
\end{aligned}
$$

Report (9) is computed for different $m_{i}$ values $\left(m_{i}=1,2\right.$, $\left.\ldots, m_{i \max }\right)$. When the Determinant Report value $\mathrm{DR}_{i}\left(m_{i}\right)$ presents an important increase over the previous value $\mathrm{DR}_{i}\left(m_{i}-1\right)$, the value $m_{i}$ corresponds to the specific order of the considered system.

As a result, the $\mathrm{DR}_{i}$ procedure can be described as

$$
\begin{aligned}
& q_{i}^{T}\left(m_{i}\right)=\left[y_{i}(k-1) u_{i}(k-1) u_{i}^{p_{1}}(k-1) u_{j}(k-1)\right. \\
& \cdot u_{j}^{p_{2}}(k-1) y_{j}(k-1) y_{j}^{p_{3}}(k-1) \cdots y_{i}\left(k-m_{i}\right) \\
& \cdot u_{i}\left(k-m_{i}\right) u_{i}^{p_{1}}\left(k-m_{i}\right) u_{j}\left(k-m_{i}\right) u_{j}^{p_{2}}\left(k-m_{i}\right) \\
& \left.\cdot y_{j}\left(k-m_{i}\right) y_{j}^{p_{3}}\left(k-m_{i}\right)\right],
\end{aligned}
$$

$$
\begin{aligned}
& Q_{i}\left(m_{i}\right)=\frac{1}{M_{k}} \sum_{m_{i}}^{M_{k}+m_{i}} q_{i}\left(m_{i}\right) q_{i}^{T}\left(m_{i}\right), \\
& \operatorname{DR}_{i}\left(m_{i}\right)=\left|\frac{\operatorname{det}\left[Q_{i}\left(m_{i}\right)\right]}{\operatorname{det}\left[Q_{i}\left(m_{i}+1\right)\right]}\right|, \\
& \quad \text { with } i, j=1, \ldots, N ; j \neq i, m_{i}=1,2, \ldots, m_{i m a x} .
\end{aligned}
$$

In order to clarify the developed approach and to test the performance of algorithm (11), we treat the following numerical example, which corresponds to a large-scale system constituted of two interconnected Hammerstein subsystems. Each system output is described by

$$
\begin{aligned}
y_{i}(k)= & -a_{i, 1}(k) y_{i}(k-1)-a_{i, 2}(k) y_{i}(k-2) \\
& +\alpha_{i, 1} u_{i}(k-1)+\alpha_{i, 1} b_{i, 2}(k) u_{i}(k-2) \\
& +\alpha_{i, 2} u_{i}^{2}(k-1)+\alpha_{i, 2} b_{i, 2}(k) u_{i}^{2}(k-2) \\
& +\beta_{j, 1} u_{j}(k-1)+\beta_{j, 1} b_{i j, 2}(k) u_{j}(k-2) \\
& +\beta_{j, 2} u_{j}^{2}(k-1)+\beta_{j, 2} b_{i j, 2}(k) u_{j}^{2}(k-2) \\
& +e_{i}(k)+c_{i, 1} e_{i}(k-1)
\end{aligned}
$$

with

$$
\begin{aligned}
a_{1,1}(k) & =-0.85+0.06 \sin (0.3 k), \\
a_{1,2}(k) & =0.37+0.05 \cos (0.3 k), \\
b_{1,2}(k) & =0.66+0.05 \sin (0.2 k), \\
b_{12,2}(k) & =0.28+0.03 \sin (0.4 k), \\
\alpha_{1,1} & =0.35, \\
\alpha_{1,2} & =0.2, \\
\beta_{2,1} & =0.43, \\
\beta_{2,2} & =0.3, \\
c_{1,1} & =0.24, \\
a_{2,1}(k) & =-0.82+0.05 \cos (0.3 k), \\
a_{2,2}(k) & =0.4+0.03 \sin (0.4 k), \\
b_{2,2}(k) & =0.58+0.03 \sin (0.2 k), \\
b_{21,2}(k) & =0.23+0.03 \sin (0.2 k), \\
\alpha_{2,1} & =0.43, \\
\alpha_{2,2} & =0.3, \\
\beta_{1,1} & =0.35, \\
\beta_{1,2} & =0.2, \\
c_{1,2} & =0.35 .
\end{aligned}
$$

In this simulation example, the input $u_{i}(k), i=1,2$, that applied to the interconnected Hammerstein system $S_{i}$ 

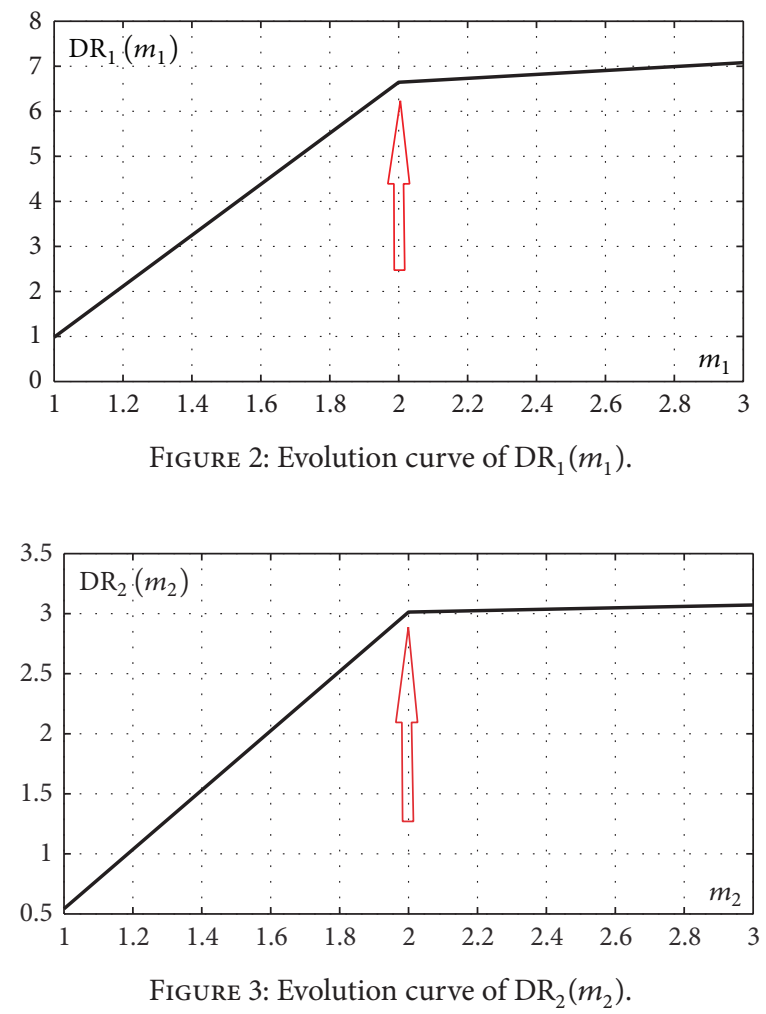

is a high level pseudorandom binary sequence $[-1.5,+1.5]$. In addition, the noise sequences $\left\{e_{i}(k), i=1,2\right\}$ are uncorrelated with all input-output signals of the considered system, their variance values are equal to $\sigma_{1}^{2}=0.0943$ and $\sigma_{2}^{2}=0.0879$, respectively, and the measurements number is selected as $M_{k}=1, \ldots, 600$.

We remark that the evolution curves of the different determinant reports, as delineated in Figures 2 and 3, admit a specific jumping in $m_{i}=2$. As a result, we can conclude that the model order is equal to 2 for each interconnected subsystem $S_{i}$. Therefore, these results indicate the performance and the efficiency of the developed algorithm (11) for the order estimate of a large-scale interconnected nonlinear system described by the Hammerstein model (1).

It should be mentioned that this approach is used to limit the number of possible model orders before starting the parameters estimation. This method gives the correct model order independently of the number of measurements because from all the obtained values of $\mathrm{DR}_{i}\left(m_{i}\right)$ only $\mathrm{DR}_{i}\left(n_{i}\right)$ of the exact order model presents a considerable step. For a system with small noise level, this test still indicates the correct order. So far, this method seems sufficient to be applied for a process with uncorrelated and high disturbances. Nevertheless, in real applications, when the noise is corrupted with all input and/or output signals, this technique becomes not suitable to determine the correct order model. In this sense, other tests methods can be used, which are developed in the literature to estimate the model order for simple systems, like the condition number method, test of signals errors, statistical $F$-test, polynomial approach, test for normality, and so forth. These techniques could be applied in combination with the parameter estimation methods. Let us note that the determinant ratio approach proves to be very robust under all working conditions because, with this test, it is possible to reduce the possibilities of the model order, as well as save a lot of computing time.

Note that the practical value of these different testing order approaches depends on the purpose and further application of the estimated model. However, it must be considered that the use of one testing approach alone may produce a wrong order model; therefore, the use of various methods together can elicit the correct order model with important accuracy.

3.2. Time-Delay Estimation. In reality, there are no physical systems without delay. The time delay as an active research in automatic control and signal processing is widely used in several industrial applications like chemical processes, energy processes, communication processes, and so on. However, it is assumed to be negligible in several researches, in order to simplify the study. To overcome this assumption, a variety of algorithms are introduced into the time-delay estimation, in order to improve the precision and the convergence in the modeling, identification, and control processes [23-28]. The time-delay identification is a greatly studied problem with several works in the literature. In this instance, different discrete-time and continuous-time techniques for time-delay estimation were proposed in control process and signal processing. Some typical approaches are described: Zhang and Li proposed a time-delay identification approach based on cross-correlation technology in signal processes [25], Zheng and Feng developed a time-delay estimation algorithm 


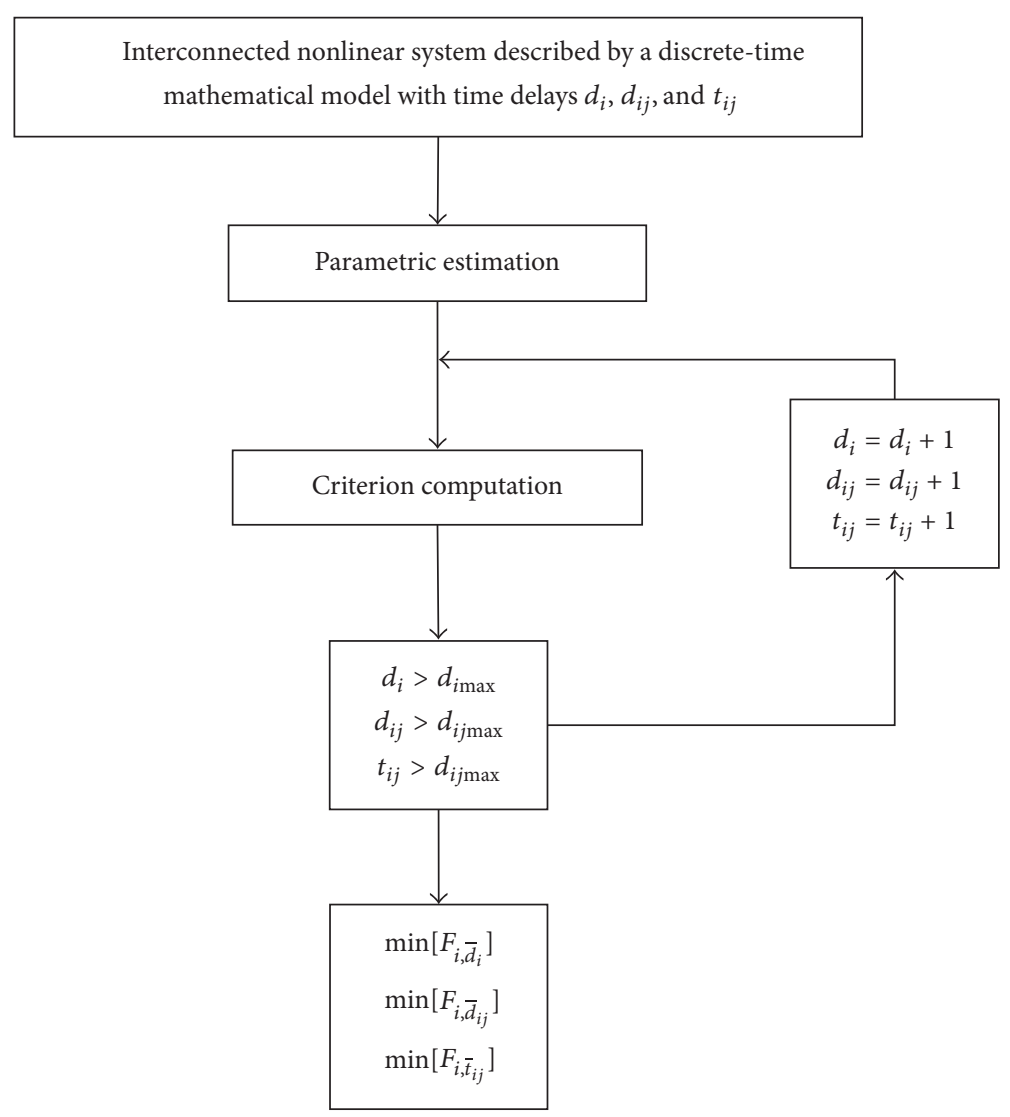

FIGURE 4: General procedure of the delay estimation.

based on correlation analysis [26], Gao et al. presented an iterative approach for time-delay estimation based on the output error between the process output and the predictive model $[27,28]$, and so forth.

In what follows, we propose an iterative approach incorporated with a Recursive Extended Least Squares estimator and based on the prediction error method, in order to estimate simultaneously the time delays and the parameters of a large-scale interconnected nonlinear process, which is modeled by (1).

The general procedure of time-delay estimation, as shown in Figure 4, is based on the computation of the criteria $F_{i, \bar{d}_{i}}$, $F_{i, \bar{d}_{i j}}$, and $F_{i, \bar{t}_{i j}}$ for each of the time delays $d_{i}, d_{i j}$, and $t_{i j}$. The minimum value of each criterion corresponds to the exact estimated delay.

To apply this procedure, let us consider a large-scale system decomposed into $N$ interconnected nonlinear subsystems and described by the mathematical model (1). Each system output can be written in the following manner:

$$
\begin{aligned}
y_{i}(k)= & \frac{B_{i r_{1}}^{*}\left(q^{-1}, k\right)}{A_{i}\left(q^{-1}, k\right)} u_{i}^{r_{1}}(k) \\
& +\frac{\sum_{j=1, j \neq i}^{N} B_{i j r_{2}}^{*}\left(q^{-1}, k\right)}{A_{i}\left(q^{-1}, k\right)} u_{j}^{r_{2}}(k)
\end{aligned}
$$

$$
\begin{aligned}
& +\frac{\sum_{j=1, j \neq i}^{N} A_{i j r_{3}}^{*}\left(q^{-1}, k\right)}{A_{i}\left(q^{-1}, k\right)} y_{j}^{r_{3}}(k) \\
& +\frac{C_{i}\left(q^{-1}\right)}{A_{i}\left(q^{-1}, k\right)} e_{i}(k),
\end{aligned}
$$

where

$$
\begin{aligned}
& B_{i r_{1}}^{*}\left(q^{-1}, k\right)=\sum_{r_{1}=1}^{p_{1}} q^{-d_{i}} B_{i}\left(q^{-1}, k\right) \alpha_{i, r_{1}}, \\
& B_{i j r_{2}}^{*}\left(q^{-1}, k\right)=\sum_{r_{2}=1}^{p_{2}} q^{-d_{i j}} B_{i j}\left(q^{-1}, k\right) \beta_{j, r_{2}}, \\
& A_{i j r_{3}}^{*}\left(q^{-1}, k\right)=\sum_{r_{3}=1}^{p_{3}} q^{-t_{i j}} A_{i j}\left(q^{-1}, k\right) \gamma_{j, r_{3}},
\end{aligned}
$$

with

$$
\begin{aligned}
B_{i r_{1}}^{*}\left(q^{-1}, k\right)= & b_{i 1,1}^{*}(k) q^{-1}+\cdots \\
& +b_{i p_{1}, n_{i}+d_{i \max }}^{*}(k) q^{-\left(n_{i}+d_{i \max }\right)}, \\
B_{i j r_{2}}^{*}\left(q^{-1}, k\right)= & b_{i j 1,1}^{*}(k) q^{-1}+\cdots \\
& +b_{i j p_{2}, n_{i}+d_{i j \max }}^{*}(k) q^{-\left(n_{i}+d_{i j \max }\right)},
\end{aligned}
$$




$$
\begin{aligned}
A_{i j r_{3}}^{*}\left(q^{-1}, k\right)= & 1+a_{i j 1,1}^{*}(k) q^{-1}+\cdots \\
& +a_{i j p_{3}, n_{i}+t_{i j \max }}^{*}(k) q^{-\left(n_{i}+t_{i j \max }\right)},
\end{aligned}
$$

in which the delays $d_{i \max }, d_{i j \max }$, and $t_{i j \max }$ correspond to the superior ones which are selected a priori from the real delays $\left(r_{t}=1, \ldots, p_{t}, t=1,2,3\right)$.

Thus, the output $y_{i}(k)$ can be expressed in the following developed form:

$$
\begin{aligned}
y_{i}(k)= & -a_{i, 1}(k) y_{i}(k-1)-\cdots-a_{i, n_{i}}(k) y_{i}\left(k-n_{i}\right) \\
& +b_{i 1,1}^{*}(k) u_{i}(k-1)+b_{i 1,2}^{*}(k) u_{i}(k-2)+\cdots \\
& +b_{i 1, n_{i}+d_{i \max }}^{*}(k) u_{i}\left(k-n_{i}-d_{i \max }\right)+\cdots \\
& +b_{i p_{1}, 1}^{*}(k) u_{i}^{p_{1}}(k-1)+b_{i p_{1}, 2}^{*}(k) u_{i}^{p_{1}}(k-2) \\
& +\cdots+b_{i p_{1}, n_{i}+d_{i \max }}^{*}(k) u_{i}^{p_{1}}\left(k-n_{i}-d_{i \max }\right) \\
& +b_{i j 1,1}^{*}(k) u_{j}(k-1)+b_{i j 1,2}^{*}(k) u_{j}(k-2) \\
& +\cdots+b_{i j 1, n_{i}+d_{i j \max }}^{*}(k) u_{j}\left(k-n_{i}-d_{i j \max }\right) \\
& +\cdots+b_{i j p_{2}, 1}^{*}(k) u_{j}^{p_{2}}(k-1) \\
& +b_{i j p_{2}, 2}^{*}(k) u_{j}^{p_{2}}(k-2)+\cdots \\
& +b_{i j p_{2}, n_{i}+d_{i j \max }}^{*}(k) u_{j}^{p_{2}}\left(k-n_{i}-d_{i j \max }\right) \\
& +a_{i j 1,1}^{*}(k) y_{j}(k-1)+a_{i j 1,2}^{*}(k) y_{j}(k-2) \\
& +\cdots+a_{i j 1, n_{i}+t_{i j \max }}(k) y_{j}\left(k-n_{i}-t_{i j \max }\right) \\
& +\cdots+a_{i j p_{3}, 1}^{*}(k) y_{j}^{p_{3}}(k-1) \\
& +a_{i j p_{3}, 2}^{*}(k) y_{j}^{p_{3}}(k-2)+\cdots \\
& +a_{i j p_{3}, n_{i}+t_{i j \max }}^{*}(k) y_{j}^{p_{3}}\left(k-n_{i}-t_{i j \max }\right) \\
& +c_{i, 1} e_{i}(k-1)+\cdots+c_{i, n_{i}} e_{i}\left(k-n_{i}\right)+e_{i}(k) .
\end{aligned}
$$

Equivalently, its matrix form is as follows:

$$
y_{i}(k)=\theta_{i}^{* T}(k) \psi_{i}^{*}(k)+e_{i}(k),
$$

where the parameters vector $\theta_{i}^{* T}(k)$ and the information vector $\psi_{i}^{*}(k)$ are defined as

$$
\begin{aligned}
& \theta_{i}^{* T}(k)=\left[a_{i, 1}(k), \ldots, a_{i, n_{i}}(k), b_{i 1,1}^{*}(k), \ldots, b_{i 1, n_{i}+d_{\text {max }}}^{*}(k),\right. \\
& \quad \ldots, b_{i p_{1}, 1}^{*}(k), \ldots, b_{i p_{1}, n_{i}+d_{i \max }}^{*}(k), b_{i j 1,1}^{*}(k), \ldots, \\
& b_{i j 1, n_{i}+d_{i j \max }^{*}}^{*}(k), \ldots, b_{i j p_{2}, 1}^{*}(k), \ldots, b_{i j p_{2}, n_{i}+d_{i j \max }^{*}}^{*}(k), \\
& a_{i j 1,1}^{*}(k), \ldots, a_{i j 1, n_{i}+t_{i j \max }}^{*}(k), \ldots, a_{i j p_{3}, 1}^{*}(k), \ldots \\
& \left.a_{i j p_{3}, n_{i}+t_{i j \max }}^{*}(k), c_{i, 1}, \ldots, c_{i, n_{i}}\right]
\end{aligned}
$$

$$
\begin{gathered}
\psi_{i}^{* T}(k)=\left[-y_{i}(k-1), \ldots,-y_{i}\left(k-n_{i}\right), u_{i}(k-1),\right. \\
\ldots, u_{i}\left(k-n_{i}-d_{i \max }\right), \ldots, u_{i}^{p_{1}}(k-1), \ldots, \\
u_{i}^{p_{1}}\left(k-n_{i}-d_{i \max }\right), u_{j}(k-1), \ldots, \\
u_{j}\left(k-n_{i}-d_{i j \max }\right), \ldots, u_{j}^{p_{2}}(k-1), \ldots, \\
u_{j}^{p_{2}}\left(k-n_{i}-d_{i j \max }\right), y_{j}(k-1), \ldots, \\
y_{j}\left(k-n_{i}-t_{i j \max }\right), \ldots, y_{j}^{p_{3}}(k-1), \ldots, \\
\left.y_{j}^{p_{3}}\left(k-n_{i}-t_{i j \max }\right), e_{i}(k-1), \ldots, e_{i}\left(k-n_{i}\right)\right] .
\end{gathered}
$$

The previous vectors, given by (19) and (20), are used in a recursive algorithm of parameter estimation, named Recursive Extended Least Squares with Varying Time (RELSVT) delay estimator. As a result, fewer steps are required to estimate the time delays.

Step 1.

(i) Determine the maximum values of $b_{i, d_{i \max }}^{*}, b_{i j, d_{i j \max }}^{*}$, and $a_{i j, t_{i j \max }}^{*}$ in order to frame the real values of the delays $d_{i}, d_{i j}$, and $t_{i j}$ :

$$
\begin{aligned}
b_{i, d_{\text {max }}}^{*} & =\left\{\left|b_{i, r}^{*}\right| ; r=1,2, \ldots, n_{i}+d_{i \max }\right\}, \\
b_{i j, d_{i j \max }}^{*} & =\left\{\left|b_{i j, r}^{*}\right| ; r=1,2, \ldots, n_{i}+d_{i j \max }\right\}, \\
a_{i j, t_{i j \max }}^{*} & =\left\{\left|a_{i j, r}^{*}\right| ; r=1,2, \ldots, n_{i}+t_{i j \max }\right\} .
\end{aligned}
$$

(ii) Verify the following relationships:

$$
\begin{aligned}
& b_{i, k_{1}}^{*}=0 \text { for } k_{1}=1, \ldots, d_{i}, \\
& b_{i, k_{2}}^{*}=b_{i, k_{2}-d_{i}}^{*} \text { for } k_{2}=d_{i}+1, \ldots, n_{i}+d_{i}, \\
& b_{i, k_{3}}^{*}=0 \text { for } k_{3}=n_{i}+d_{i}+1, \ldots, n_{i}+d_{i \max }, \\
& b_{i j, k_{1}}^{*}=0 \text { for } k_{1}=1, \ldots, d_{i j}, \\
& b_{i j, k_{2}}^{*}=b_{i j, k_{2}-d_{i j}}^{*} \text { for } k_{2}=d_{i j}+1, \ldots, n_{i}+d_{i j}, \\
& b_{i j, k_{3}}^{*}=0 \text { for } k_{3}=n_{i}+d_{i j}+1, \ldots, n_{i}+d_{i j \max }, \\
& a_{i j, k_{1}}^{*}=0 \text { for } k_{1}=1, \ldots, t_{i j}, \\
& a_{i j, k_{2}}^{*}=a_{i j, k_{2}-t_{i j}}^{*} \text { for } k_{2}=t_{i j}+1, \ldots, n_{i}+t_{i j}, \\
& a_{i j, k_{3}}^{*}=0 \text { for } k_{3}=n_{i}+t_{i j}+1, \ldots, n_{i}+t_{i j \max } .
\end{aligned}
$$

Thereby, the real delay satisfies the relation given as

$$
\begin{aligned}
& 0 \leq \widehat{d}_{i} \leq d_{i \max }-1, \\
& 0 \leq \widehat{d}_{i j} \leq d_{i j \max }-1, \\
& 0 \leq \widehat{t}_{i j} \leq t_{i j \max }-1 .
\end{aligned}
$$


Step 2. Compute the errors functions $F_{i, \bar{d}_{i}}, F_{i, \bar{d}_{i j}}$, and $F_{i, \bar{t}_{i j}}$ starting from

$$
\begin{aligned}
& F_{i, \bar{d}_{i}}(k)=\sum_{k=0}^{M_{k}} \widehat{G}_{i, \bar{d}_{i}}^{2}(k) ; \quad \bar{d}_{i}=0, \ldots, d_{i \max }-1, \\
& F_{i, \bar{d}_{i j}}(k)=\sum_{k=0}^{M_{k}} \widehat{H}_{i, \bar{d}_{i j}}^{2}(k) ; \quad \bar{d}_{i j}=0, \ldots, d_{i j \max }-1, \\
& F_{i, \bar{t}_{i j}}(k)=\sum_{k=0}^{M_{k}} \widehat{K}_{i, \bar{t}_{i j}}^{2}(k) ; \quad \bar{t}_{i j}=0, \ldots, t_{i j \max }-1,
\end{aligned}
$$

where $\widehat{G}_{i, \bar{d}_{i}}(k), \widehat{H}_{i, \bar{d}_{i j}}(k)$, and $\widehat{K}_{i, \bar{t}_{i j}}(k)$ are given as follows. If $\bar{d}_{i}=0$, then

$$
\begin{aligned}
& \widehat{G}_{i, 0}(k)=0 \quad \text { for } k=1, \ldots, n_{i}, \\
& \widehat{G}_{i, 0}(k)=\widehat{b}_{i, k}^{*}-\sum_{r=1}^{n_{i}} \widehat{a}_{i, r} \widehat{G}_{i, 0}(k-r) \\
& \quad \text { for } k=n_{i}+1, \ldots, n_{i}+d_{i \max }, \\
& \widehat{G}_{i, 0}(k)=-\sum_{r=1}^{n_{i}} \widehat{a}_{i, r} \widehat{G}_{i, 0}(k-r) \\
& \quad \text { for } k=n_{i}+d_{i \max }+1, \ldots, M_{k} .
\end{aligned}
$$

If $\bar{d}_{i} \geq 1$, then

$$
\begin{aligned}
& \widehat{G}_{i, \bar{d}_{i}}(k)=0 \quad \text { for } k=0, \\
& \widehat{G}_{i, \bar{d}_{i}}(k)=\widehat{b}_{i, k}^{*}-\sum_{r=1}^{n_{i}} \widehat{a}_{i, r} \widehat{G}_{i, \bar{d}_{i}}(k-r) \quad \text { for } k=2, \ldots, \bar{d}_{i}, \\
& \widehat{G}_{i, \bar{d}_{i}}(k)=-\sum_{r=1}^{n_{i}} \widehat{a}_{i, r} \widehat{G}_{i, \bar{d}_{i}}(k-r)
\end{aligned}
$$$$
\text { for } k=\bar{d}_{i}+1, \ldots, n_{i}+\bar{d}_{i} \text {, }
$$$$
\begin{aligned}
& \widehat{G}_{i, \bar{d}_{i}}(k)=\widehat{b}_{i, k}^{*}-\sum_{r=1}^{n_{i}} \widehat{a}_{i, r} \widehat{G}_{i, \bar{d}_{i}}(k-r) \\
& \qquad \text { for } k=n_{i}+\bar{d}_{i}+1, \ldots, n_{i}+d_{i \max },
\end{aligned}
$$$$
\widehat{G}_{i, \bar{d}_{i}}(k)=-\sum_{r=1}^{n_{i}} \widehat{a}_{i, r} \widehat{G}_{i, \bar{d}_{i}}(k-r)
$$

$$
\begin{array}{r}
\widehat{H}_{i, 0}(k)=-\sum_{r=1}^{n_{i}} \widehat{a}_{i, r} \widehat{H}_{i, 0}(k-r) \\
\text { for } k=n_{i}+d_{i j \max }+1, \ldots, M_{k} .
\end{array}
$$

If $\bar{d}_{i j} \geq 1$, then

$$
\begin{aligned}
& \widehat{H}_{i, \bar{d}_{i j}}(k)=0 \quad \text { for } k=0, \\
& \widehat{H}_{i, \bar{d}_{i j}}(k)=\widehat{b}_{i j, k}^{*}-\sum_{r=1}^{n_{i}} \widehat{a}_{i, r} \widehat{H}_{i, \bar{d}_{i j}}(k-r)
\end{aligned}
$$

for $k=2, \ldots, \bar{d}_{i j}$

$$
\begin{aligned}
& \widehat{H}_{i, \bar{d}_{i j}}(k)=-\sum_{r=1}^{n_{i}} \widehat{a}_{i, r} \widehat{H}_{i, \bar{d}_{i j}}(k-r) \\
& \text { for } k=\bar{d}_{i j}+1, \ldots, n_{i}+\bar{d}_{i j},
\end{aligned}
$$

$$
\widehat{H}_{i, \bar{d}_{i j}}(k)=\widehat{b}_{i j, k}^{*}-\sum_{r=1}^{n_{i}} \widehat{a}_{i, r} \widehat{H}_{i, \bar{d}_{i j}}(k-r)
$$

for $k=n_{i}+\bar{d}_{i j}+1, \ldots, n_{i}+d_{i j \max }$,

$$
\widehat{H}_{i, \bar{d}_{i j}}(k)=-\sum_{r=1}^{n_{i}} \widehat{a}_{i, r} \widehat{H}_{i, \bar{d}_{i j}}(k-r)
$$

$$
\text { for } k=n_{i}+d_{i j \max }+1, \ldots, M_{k} \text {. }
$$

If $\bar{t}_{i j}=0$, then

$$
\begin{aligned}
& \widehat{K}_{i, 0}(k)=0 \quad \text { for } k=1, \ldots, n_{i}, \\
& \widehat{K}_{i, 0}(k)=\widehat{a}_{i j, k}^{*}-\sum_{r=1}^{n_{i}} \widehat{a}_{i, r} \widehat{K}_{i, 0}(k-r) \\
& \quad \text { for } k=n_{i}+1, \ldots, n_{i}+t_{i j \max }, \\
& \widehat{K}_{i, 0}(k)=-\sum_{r=1}^{n_{i}} \widehat{a}_{i, r} \widehat{K}_{i, 0}(k-r)
\end{aligned}
$$

for $k=n_{i}+t_{i j \max }+1, \ldots, M_{k}$. 
If $\bar{t}_{i j} \geq 1$, then

$$
\begin{aligned}
& \widehat{K}_{i, \bar{t}_{i j}}(k)=0 \quad \text { for } k=0, \\
& \widehat{K}_{i, \bar{t}_{i j}}(k)=\widehat{a}_{i j, k}^{*}-\sum_{r=1}^{n_{i}} \widehat{a}_{i, r} \widehat{K}_{i, \bar{t}_{i j}}(k-r) \\
& \quad \text { for } k=2, \ldots, \bar{t}_{i j}, \\
& \widehat{K}_{i, \bar{t}_{i j}}(k)=-\sum_{r=1}^{n_{i}} \widehat{a}_{i, r} \widehat{K}_{i, \bar{t}_{i j}}(k-r) \\
& \text { for } k=\bar{t}_{i j}+1, \ldots, n_{i}+\bar{t}_{i j},
\end{aligned}
$$

$$
\widehat{K}_{i, \bar{t}_{i j}}(k)=\widehat{a}_{i j, k}^{*}-\sum_{r=1}^{n_{i}} \widehat{a}_{i, r} \widehat{K}_{i, \bar{t}_{i j}}(k-r)
$$$$
\text { for } k=n_{i}+\bar{t}_{i j}+1, \ldots, n_{i}+t_{i j \max },
$$$$
\widehat{K}_{i, \bar{t}_{i j}}(k)=-\sum_{r=1}^{n_{i}} \widehat{a}_{i, r} \widehat{K}_{i, \bar{t}_{i j}}(k-r)
$$

$$
\text { for } k=n_{i}+t_{i j \max }+1, \ldots, M_{k} \text {. }
$$

Step 3.

(i) Determine the estimated delay that satisfies

$$
\begin{aligned}
& F_{i, \widehat{d}_{i}}=\min \left\{F_{i, \bar{d}_{i}}(\mathrm{k}) ; \bar{d}_{i}=0, \ldots, d_{i \max }-1\right\}, \\
& F_{i, \widehat{d}_{i j}}=\min \left\{F_{i, \bar{d}_{i j}}(k) ; \bar{d}_{i j}=0, \ldots, d_{i j \max }-1\right\}, \\
& F_{i, \bar{t}_{i j}}=\min \left\{F_{i, \bar{t}_{i j}}(k) ; \bar{t}_{i j}=0, \ldots, t_{i j \max }-1\right\} .
\end{aligned}
$$

The minimum values of $F_{i, \bar{d}_{i}}, F_{i, \bar{d}_{i j}}$, and $F_{i, \bar{t}_{i j}}$ indicate, respectively, the exact time delays $d_{i}, d_{i j}$, and $t_{i j}$ of the process.

To validate the proposed approach of time-delay estimation, let us consider the previous numerical example with $d_{1}=1$ and $d_{2}=2$. Figures 5 and 6 represent the evolution curves of $F_{i, \bar{d}_{i}}, i=1,2$, for each interconnected nonlinear subsystem.

According to the evolution curves of $F_{1, \bar{d}_{1}}$ and $F_{2, \bar{d}_{2}}$, it can be remarked that $\widehat{d}_{1}=1$ and $\widehat{d}_{2}=2$. These simulation results prove the validity and feasibility of the proposed algorithm for time-delay estimation of the large-scale interconnected nonlinear processes.

It can be remarked that the estimated delays depend on the estimated parameters of the model. Therefore, (31) is added to an Extended Least Squares algorithm to estimate simultaneously the parameters and the time delays of the model. Note that computer simulations show that discrete solutions properties of the delays equations make them robust to estimation errors in the estimate of parameters vector (19) and the presence of Gaussian-distributed noise. If the parameters are correctly estimated and no disturbances exist,
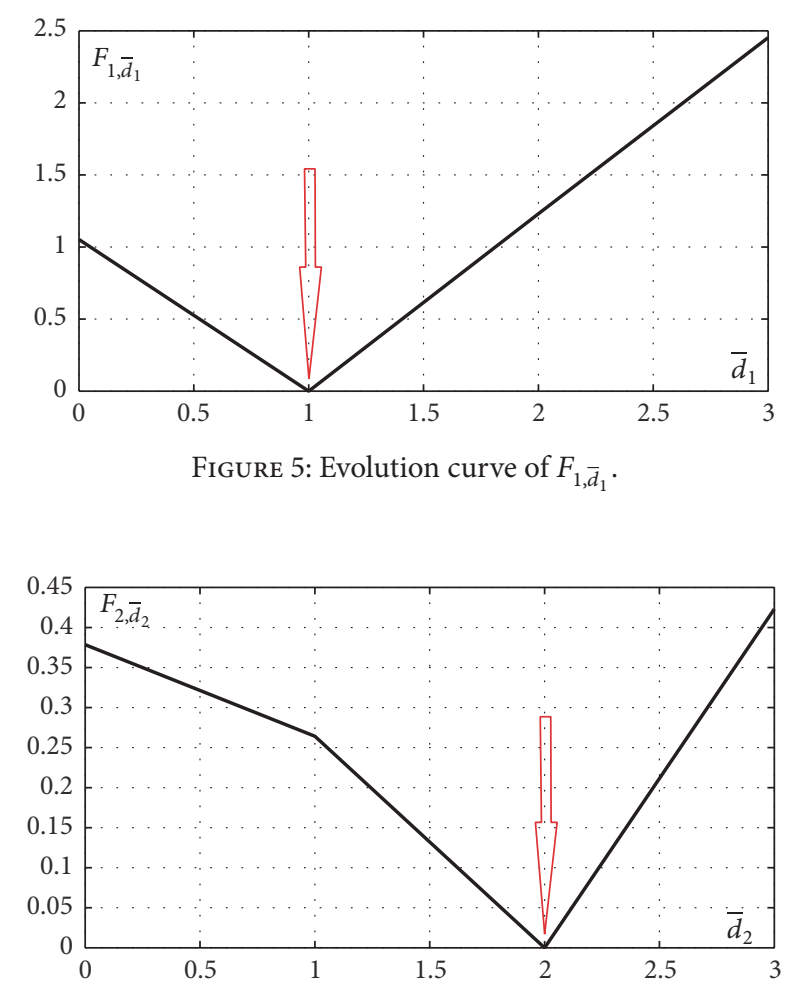

FIGURE 6: Evolution curve of $F_{2, \bar{d}_{2}}$.

(31) will have a zero minimum. If there is a small estimation error and small noise level, (31) is still enough to identify the delays correctly. As the estimation error and the level noise increase, the delays estimation can be conducted using the maximum likelihood algorithm in the estimation of the process parameters and based on (31) (the maximum likelihood estimator is more robust than the Recursive Extended Least Squares algorithm in the convergence sense). It should be mentioned that (31) can be added easily to any recursive estimator. The implementation of this equation requires minimum storage and computation time as they contain multiplications and additions.

However, in realistic applications, the corrupting noise is correlated with input and/or output signals, so this makes the estimates of the parameters asymptotically biased, as well as a wrong estimated time delay. In this case, we can use other techniques to estimate the time delays and the parameters of the model, such as the correlation analysis, the crosscorrelation method, and generalized $l p$ norm estimation. In conclusion, a priori information of noise distribution is necessary in order to develop an efficient estimation.

In the identification systems, two main problems must be considered. The first one is the identification of the time delay, and the second one is the identification of the parameters. In this case, the time-delay estimation problem is a great research axis with several references dealing with several approaches for time-delay estimation. These approaches can be classified into four classes. The first class denotes the time-delay approximation approach (time domain, frequency domain, and Laguerre domain). In this case, the time delay is 
estimated from an approximation of the model. The second class represents the time-delay parameter method (one step, two steps, and sampling method). In this case, the time delay and the parameters of the model are identified alternatively, simultaneously, or using the sampling system. The third class is the area and moments approaches. In this event, we estimate firstly the step or the impulse response. After that, we identify the time delay. The last class represents the high-order statistics approach. The main disadvantage of these techniques is that parameters estimation is required on each iteration step of time-delay estimation, and this makes the implementation difficult. Furthermore, it can be indicated that there is not an efficient solution to the timedelay problem. The general agreement is on which method is the best. Thereby, a comparative study of these techniques for time-delay estimation of large-scale interconnected nonlinear systems will be investigated in the future research.

\section{Conclusions}

Two extended discrete-time structural estimation methods, which are applied for estimating the time delay and the order of a dynamical process, are developed. We have particularly focused on the class of large-scale time-varying stochastic systems constituted by various interconnected Hammerstein structures. These systems are described by discrete-time stochastic Hammerstein mathematical models with unknown time-varying parameters.

The main research work developed in this article is divided into two important parts. The first part formulated the problem of the order estimation for the class of large-scale interconnected nonlinear systems, using the determinant ratio test $\mathrm{DR}_{i}$. The second part proposed an extended iterative procedure, which permits estimating the time delays of the considered system.

These developed approaches are validated by treating two numerical simulation examples of a large-scale system composed of two interconnected Hammerstein structures. The obtained results prove the performance and the feasibility of the proposed methods in spite of the presence of interactions signals, Gaussian distribution noise, and parameters variations.

\section{Conflicts of Interest}

The authors declare that there are no conflicts of interest regarding the publication of this paper.

\section{References}

[1] M. Hamdy, "State observer based dynamic fuzzy logic system for a class of SISO nonlinear systems," International Journal of Automation and Computing, vol. 10, no. 2, pp. 118-124, 2013.

[2] H. Hu and D. Zhao, "Decentralized Ho control for uncertain interconnected systems of neutral type via dynamic output feedback," Abstract and Applied Analysis, Article ID 989703, Art. ID 989703, 11 pages, 2014.
[3] G. B. Koo, J. B. Park, and Y. H. Joo, "Decentralized fuzzy observer-based output-feedback control for nonlinear largescale systems: an LMI approach," IEEE Transactions on Fuzzy Systems, vol. 22, no. 2, pp. 406-419, 2014.

[4] N. Chen, Y. Liu, B. Liu, and W. Gui, "Parametric absolute stabilization for interconnected Lurie time-delay systems with polytopic uncertainty," Asian Journal of Control, vol. 16, no. 1, pp. 225-232, 2014.

[5] B.-C. Zheng and G.-H. Yang, "Decentralized sliding mode quantized feedback control for a class of uncertain largescale systems with dead-zone input," Nonlinear Dynamics. An International Journal of Nonlinear Dynamics and Chaos in Engineering Systems, vol. 71, no. 3, pp. 417-427, 2013.

[6] S. Nagai and H. Oya, "Synthesis of decentralized variable gain robust controllers for large-scale interconnected systems with structured uncertainties," Journal of Control Science and Engineering, Article ID 848465, 10 pages, 2014.

[7] S. Nagai and H. Oya, "Synthesis of decentralized variable gain robust controllers with guaranteed $\mathscr{L}_{2}$ gain performance for a class of uncertain large-scale interconnected systems," Journal of Control Science and Engineering, Article ID 342867, 11 pages, 2015.

[8] H. Wang, B. Chen, and C. Lin, "Adaptive fuzzy decentralized control for a class of large-scale stochastic nonlinear systems," Neurocomputing, vol. 103, pp. 155-163, 2013.

[9] H. Wang, X. Liu, and K. Liu, "Robust adaptive neural tracking control for a class of stochastic nonlinear interconnected systems," IEEE Transactions on Neural Networks and Learning Systems, vol. 27, no. 3, pp. 510-523, 2016.

[10] H. Wang, Q. Zhou, X. Yang, and H. Karimi, "Robust decentralized adaptive neural control for a class of nonaffine nonlinear large-scale systems with unknown dead zones," Mathematical Problems in Engineering, Article ID 841306, 10 pages, 2014.

[11] M. Elloumi and S. Kamoun, "Parametric estimation of interconnected nonlinear systems described by input-output mathematical models," International Journal of Automation and Computing, vol. 13, no. 4, pp. 364-381, 2016.

[12] M. Elloumi and S. Kamoun, "An iterative parametric estimation method for Hammerstein large-scale systems: a simulation study of hydraulic process," International Journal of Simulation and Process Modelling, vol. 11, no. 3-4, pp. 207-219, 2016.

[13] M. Elloumi and S. Kamoun, "Design of self-tuning regulator for large-scale interconnected Hammerstein systems," Journal of Control Science and Engineering, Article ID 6769714, 13 pages, 2016.

[14] M. Elloumi and S. Kamoun, "Adaptive control scheme for large-scale interconnected systems described by Hammerstein models," Asian Journal of Control, vol. 19, no. 3, pp. 1-14, 2017.

[15] R. Haber and H. Unbehauen, "Structure identification of nonlinear dynamic systems-a survey on input/output approaches," Automatica. A Journal of IFAC, the International Federation of Automatic Control, vol. 26, no. 4, pp. 651-677, 1990.

[16] S. A. Billings, Nonlinear System Identification: NARMAX Methods in the Time and Spatio-Temporal Domains, John Wiley \& Sons, Chichester, UK, 2013.

[17] R. Haber and L. Keviczky, Nonlinear System Identification: Input-output Modelling Approach, Mathematical Modelling: Theory and Applications, Kluwer Academic Publishers, 1999.

[18] F. Ding, T. Chen, and Z. Iwai, "Adaptive digital control of Hammerstein nonlinear systems with limited output sampling," SIAM Journal on Control and Optimization, vol. 45, no. 6, pp. 2257-2276, 2007. 
[19] F. Ding, Y. Shi, and T. Chen, "Auxiliary model-based leastsquares identification methods for Hammerstein output-error systems," Systems and Control Letters, vol. 56, no. 5, pp. 373-380, 2007.

[20] F. Ding and T. Chen, "Identification of Hammerstein nonlinear ARMAX systems," Automatica. A Journal of IFAC, the International Federation of Automatic Control, vol. 41, no. 9, pp. 14791489, 2005.

[21] F. Ding, X. Wang, Q. Chen, and Y. Xiao, "Recursive least squares parameter estimation for a class of output nonlinear systems based on the model decomposition," Circuits, Systems, and Signal Processing, vol. 35, no. 9, pp. 3323-3338, 2016.

[22] S. A. Billings and S. Y. Fakhouri, "Non-linear system identification using the Hammerstein model," International Journal of Systems Science, vol. 10, no. 5, pp. 567-578, 1979.

[23] G. Ferretti, C. Maffezzoni, and R. Scattolini, "On the identifiability of the time delay with least-squares methods," Automatica. A Journal of IFAC, the International Federation of Automatic Control, vol. 32, no. 3, pp. 449-453, 1996.

[24] L. Ljung, System Identification: Theory for the User, PrenticeHall, Upper Saddle River, NJ, USA, 2nd edition, 1999.

[25] T. Zhang and Y. C. Li, "A fuzzy smith control of time-varying delay systems based on time delay identification," in Proceedings of the 2nd International Conference on Machine Learning and Cybernetics, pp. 614-619, Xi'an, China, November 2003.

[26] W. X. Zheng and C. B. Feng, "Identification of stochastic time lag systems in the presence of colored noise," Automatica. A Journal of IFAC, the International Federation of Automatic Control, vol. 26, no. 4, pp. 769-779, 1990.

[27] W. Gao, Y. Li, G. Liu, and T. Zhang, "An adaptive fuzzy smith control of time-varying processes with dominant and variable delay," in Proceedings of the 2003 American Control Conference, pp. 220-224, Denver, CO, USA, June 2003.

[28] W. Gao, M. L. Zhou, Y. C. Li, and T. Zhang, "An adaptive generalized predictive control of time-varying delay system," in Proceedings of the 2004 International Conference on Machine Learning and Cybernetics, pp. 878-881, Shanghai, China, August 2004. 


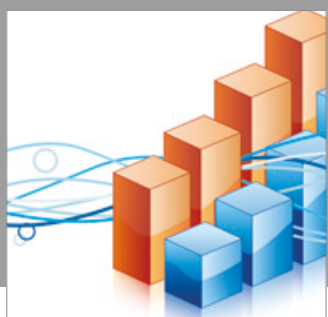

Advances in

Operations Research

vatersals

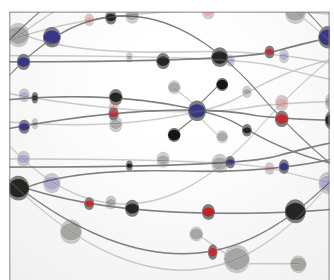

\section{The Scientific} World Journal
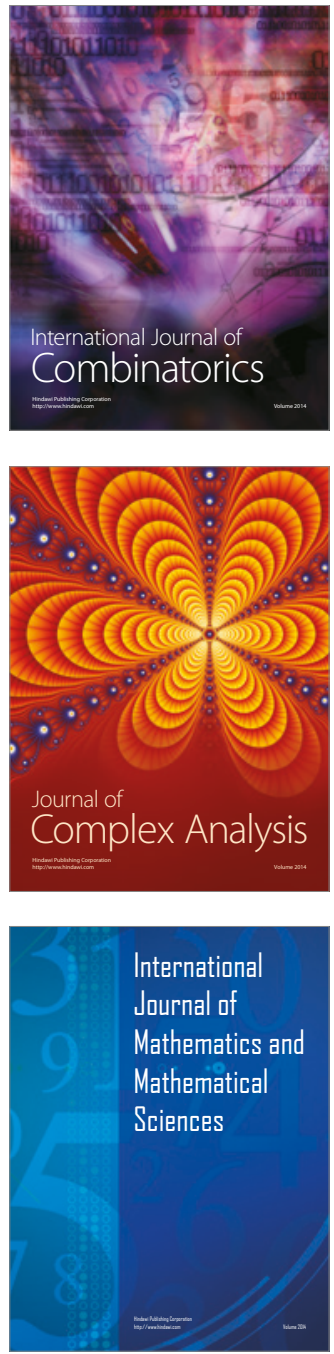
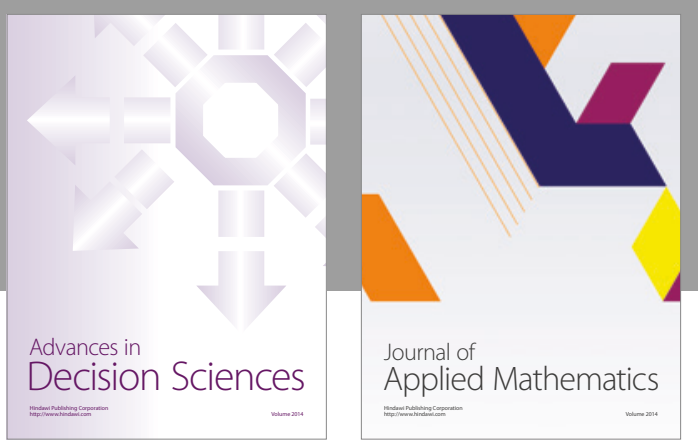

Algebra

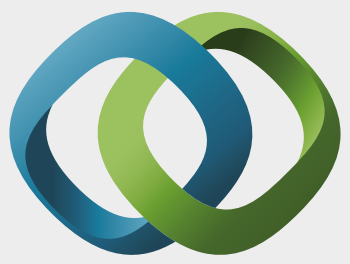

\section{Hindawi}

Submit your manuscripts at

https://www.hindawi.com
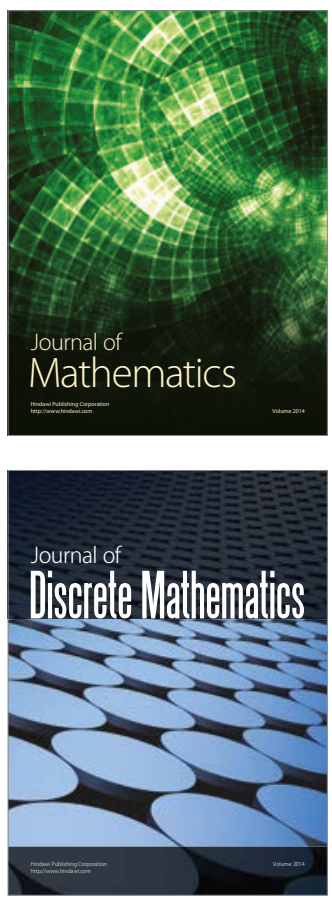

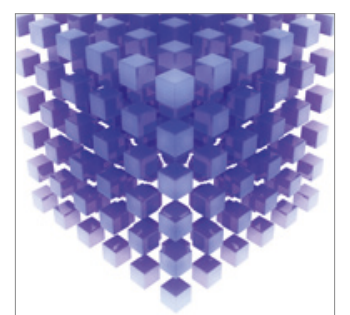

Mathematical Problems in Engineering
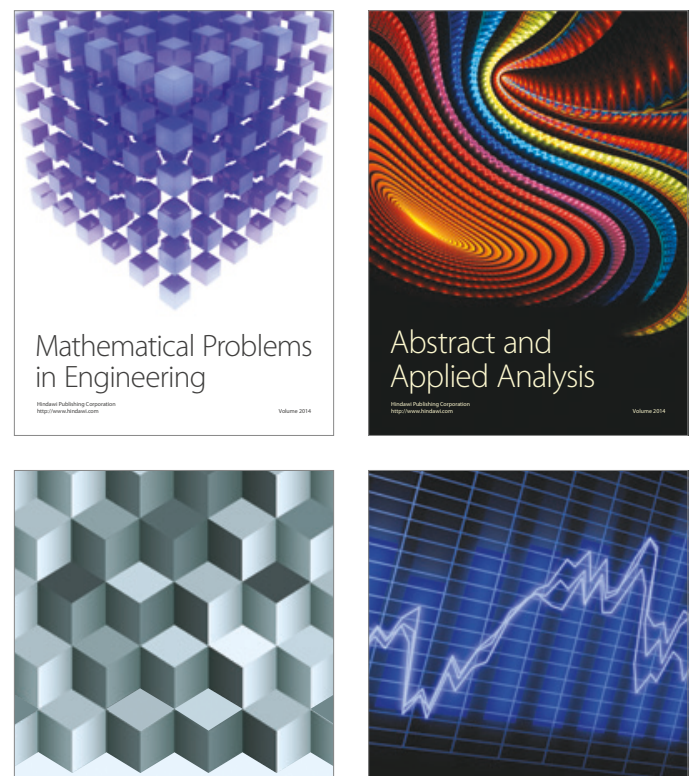

Journal of

Function Spaces

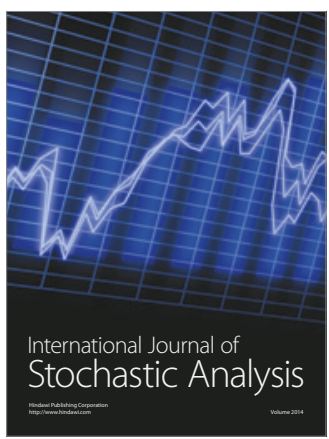

Probability and Statistics
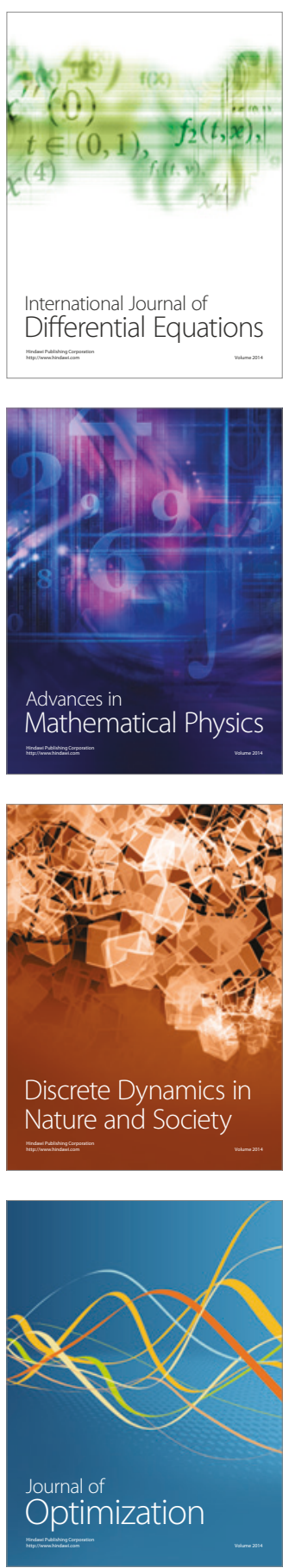